Diptesh Ghosh (Ahmedabad)

Gerard Sierksma (Groningen)

\title{
ON THE COMPLEXITY OF DETERMINING TOLERANCES FOR $\varepsilon$-OPTIMAL SOLUTIONS TO MIN-MAX COMBINATORIAL OPTIMIZATION PROBLEMS
}

Abstract. This paper studies the complexity of sensitivity analysis for optimal and $\varepsilon$-optimal solutions to general 0-1 combinatorial optimization problems with min-max objectives. Van Hoesel and Wagelmans [9] have studied the complexity of sensitivity analysis of optimal and $\varepsilon$-optimal solutions to min-sum problems, and Ramaswamy et al. [17] the complexity of sensitivity analysis of optimal solutions to min-max problems. We show that under some mild assumptions the sensitivity analysis of $\varepsilon$-optimal solutions to min-max problems is easy if and only if the original problem is easy. This result is interesting since it immediately applies to a large number of problems, and also because the technique used to prove it is different from the ones used in the related papers (for example, in [17] and [9]).

1. Introduction and motivation. Studying the effect of changes in data on optimal solutions to optimization problems is an important step in gaining insight into the problem itself, and can be carried out in a variety of ways. One can study the effect of changing a single parameter (sensitivity analysis), or several parameters simultaneously (stability analysis). One may observe the changes in an optimal solution when a parameter changes from its lowest possible to highest possible value (parametric analysis), or find the range within which a parameter must lie for the optimal solution to remain optimal (tolerance analysis). Another approach, although less popular, is to compute the set of $k$ best solutions for the problem ( $k$-best approach).

Sensitivity analysis of combinatorial optimization problems (COPs), although not as popular as that of linear optimization, has been widely studied

2000 Mathematics Subject Classification: Primary 90C31.

Key words and phrases: complexity, sensitivity analysis, min-max, 0-1 combinatorial optimization problems. 
in the last thirty years. Initial studies considered COPs as mere special cases of general integer programming problems and used the parametric analysis approach (refer, for example, to Nauss [14] for work done during that period). Later, as the body of research in this area grew, and it was reported that the sensitivity analysis for COPs may not have some of the nice properties that the sensitivity analysis of linear programming problems has (refer, for example, to Blair [1], Woeginger [22]), several dominant trends appeared. Some researchers concentrated on particular well-solved COPs, like the traveling salesperson problem (TSP). For example, in Libura [10] and van der Poort et al. [15] the tolerance approach is used to compute the amount of perturbation that would be allowed in the problem data before a currently optimal solution becomes suboptimal. In Libura et al. [13] such information is used to solve the $k$-best TSP. In Gordeev, Leontev, and Sigal [7] the stability radius of several problems is computed, one of which is the TSP. Other researchers considered sensitivity analysis problems for general integer linear programming problems or COPs (refer, for example, to Blair and Jereslow [2], Cartensen [3], Chakravarti and Wagelmans [4], Cook et al. [5], Libura [11, 12], Ramaswamy [16], Ramaswamy et al. [17], Sotskov [18, 19], Sotskov et al. [20], van Hoesel and Wagelmans [9], and Wagelmans [21]). Of these, [5], [11], [12], [18]-[20] deal with the calculations required for sensitivity analysis of these problems, while [2]-[4], [16], [17], [9], and [21] with the complexity of performing such computations. Most of the papers cited above deal with objective functions of the min-sum type. However, the min-max or bottleneck objective is considered in Gordeev and Leontev [6], [7], [9], and [21]. An extensive annotated bibliography of publications in this field after 1977 is available in Greenberg [8]. A majority of the papers in this field deal with optimal solutions to optimization problems. $\varepsilon$-optimal solutions are considered in [9], and briefly in [4], but in both in the context of min-sum objective functions. In [9] it is shown that for COPs with min-sum objectives, the complexity of sensitivity analysis of $\varepsilon$-optimal solutions is polynomial if and only if the original COP is polynomially solvable. In this paper we report the corresponding result when the objective is of the min-max type.

We deal with generic COPs. A generic COP $\Pi$ can be defined as follows. It is a collection of problem instances $\pi=(G, \mathcal{S}, z)$, where $\mathcal{S} \subseteq 2^{G}$, and $z: \mathcal{S} \rightarrow \mathbb{R}$. The set $G$ is called the ground set. Each element $e \in G$ has a cost $c_{e}$. The members of $\mathcal{S}$ are called feasible solutions, and $z$ is referred to as the objective function. Without loss of generality we will assume that $z(\emptyset)=\infty$. In general, such COPs are of two types, those with min-sum (or linear) objectives, and those with min-max objectives. A COP $\Pi$ is said to have a min-sum objective if $z(S)=\sum_{e \in S} c_{e}$, and a min-max objective if $z(S)=\max \left\{c_{e}: e \in S\right\}$ for every $S \in \mathcal{S}$ and each $\pi \in \Pi$. In this paper we refer to them as min-sum COPs and min-max COPs respectively. 
We denote by $\operatorname{OPT}(\Pi)$ the problem of finding an optimal solution $S^{*}$, i.e. a member of $\arg \min \{z(S): S \in \mathcal{S}\}$ for any given instance $\pi \in \Pi$. For any $\varepsilon>0$ we denote by $\mathrm{OPT} \varepsilon(\Pi)$ the problem of finding an $\varepsilon$-optimal solution, i.e. a member of $\left\{S: S \in \mathcal{S},\left|z(S)-z\left(S^{*}\right)\right| \leq \varepsilon\left|z\left(S^{*}\right)\right|\right\}$.

The sensitivity analysis problem $(\mathrm{SA}(\Pi))$ of a $\mathrm{COP} \Pi$ using the tolerance approach involves finding, for each parameter, upper and lower bounds within which the value of the parameter can vary for the optimal solution to remain optimal. For $\varepsilon$-optimal solutions to general COPs, this description can be extended to the definition of the following problem (see, for example, [9]).

Problem SA $\varepsilon(\Pi)$ : Sensitivity analysis of $\varepsilon$-optimal solutions of a COP $\Pi$ Input: Instance $\pi=(G, \mathcal{S}, z)$ of $\Pi, \varepsilon>0$, an $\varepsilon$-optimal solution $S^{\varepsilon}$ to $\pi$. Output: For each $e \in G$,

upper tolerance limit $\beta_{e}$

$$
=\sup \left\{\delta \in \mathbb{R}: S^{\varepsilon} \text { remains } \varepsilon \text {-optimal when } c_{e} \leftarrow c_{e}+\delta\right\},
$$

lower tolerance limit $\alpha_{e}$

$$
=\sup \left\{\delta \in \mathbb{R}: S^{\varepsilon} \text { remains } \varepsilon \text {-optimal when } c_{e} \leftarrow c_{e}-\delta\right\} .
$$

The complexity of $\mathrm{SA}(\Pi)$ and $\mathrm{SA} \varepsilon(\Pi)$ was analyzed for general min-sum COPs $\Pi$ in [9]. It was shown that both these problems are easy if and only if $\operatorname{OPT}(\Pi)$ is easy. In [17] it was shown that $\mathrm{SA} \varepsilon(\Pi)$ is easy for general min-sum COPs $\Pi$ if and only if $\mathrm{OPT}(\Pi)$ is easy.

In this paper we study the complexity aspects of $\mathrm{SA} \varepsilon(\Pi)$ for a general min-max COP $\Pi$. We cannot directly use either the results from the complexity analysis of $\mathrm{SA}(\Pi)$ for min-max COPs, or those of $\mathrm{SA} \varepsilon(\Pi)$ for min-sum COPs to derive these results. Complexity analysis for $\mathrm{SA}(\Pi)$ (for example in [17]) makes use of the fact that the solution at hand has the lowest $z$ value possible. This assumption cannot be utilized when analyzing $\varepsilon$-optimal solutions. Arguments used to analyze the complexity of $\mathrm{SA} \varepsilon(\Pi)$ for min-sum problems (for example in [9]) also do not translate directly to arguments for min-max problems. This is due to the difference in the nature of the objective functions in the two cases. If an element $e$ is a member of a solution $S$ of an instance of a min-sum COP, then any change in $c_{e}$ is reflected in $z(S)$. Hence the graph of $z(S)$ against $c_{e}$ is a straight line with slope 1 (see Figure 1(a)). However, for an instance of a min-max COP, even if an element $e$ is a member of a solution $S$, changes in $c_{e}$ affect $z(S)$ if and only if $c_{e}$ is not less than the cost of any other element in $S$. Hence the graph of $z(S)$ against $c_{e}$ has two components, one with slope 0 , and another with slope 1, as shown in Figure 1(b). This difference makes it impossible to make direct use of the techniques used, for example in [9], for sensitivity analysis of $\varepsilon$-optimal solutions to min-max COPs. 


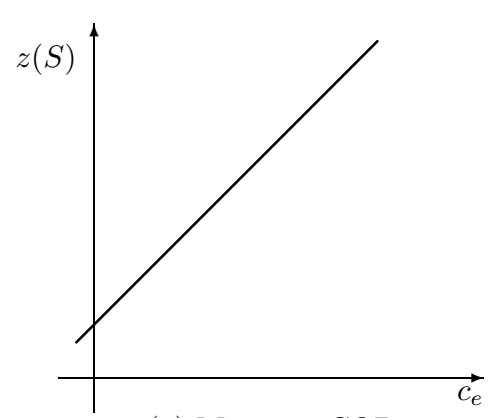

(a) Min-sum COP

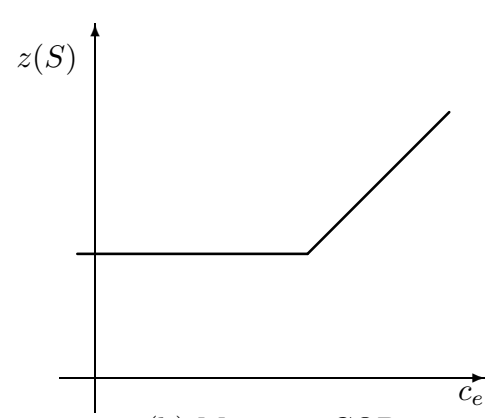

(b) Min-max COP

Fig. 1. Variation of $z(S)$ with $c_{e}$ when $e \in S$

2. Sensitivity analysis. We will use the following notation in the remainder of the paper. $\mathcal{P}$ will denote the set of polynomially solvable optimization problems. The COP at hand will be denoted by $\Pi ; \pi$ will denote an instance of $\Pi$. If the cost of any problem element of $\pi$ is changed by a cost transformation $T$, the new instance will be denoted by $\pi_{T}$. Furthermore, $S^{*}$ will denote an optimal solution to $\pi$ and $S^{\varepsilon}$ an $\varepsilon$-optimal solution. Given an element $e \in G, S^{e}$ will denote an element of the set $\{S: S \in \mathcal{S}, e \notin S\}$. Given $e \in S \in \mathcal{S}$, we call $S$ e-critical with respect to $z$ if $z(S)=c_{e}$. Given a solution $S=\left\{e_{[1]}, e_{[2]}, \ldots\right\}$ with $c_{e_{[1]}} \geq c_{e_{[2]}} \geq \ldots$, the cost of the second largest element of $S$, i.e. $c_{e_{[2]}}$, will be denoted by $c_{[2]}(S)$. If $S$ is a singleton, then $c_{[2]}(S)$ is assumed to be $\infty$. Note that $c_{[2]}(S)$ may equal $z(S)$.

Let us first assume that $\mathrm{OPT}(\Pi) \in \mathcal{P}$ and that a polynomial algorithm $\mathcal{A}$ solves any instance of $\Pi$. Note that for any $e \in G$ and any instance $\pi$ of $\Pi, S^{e}$ can be found in polynomial time by setting $c_{e} \leftarrow \infty$. In the following lemma, we are concerned with the complexity of finding the cost of a smallest second largest element in any $e$-critical solution, when $c_{e}$ is at least as large as $z\left(S^{*}\right)$.

LEMma 1. If $\operatorname{OPT}(\Pi) \in \mathcal{P}$, then given an instance $\pi$ of $\Pi$ and $e \in G$ with $c_{e} \geq z\left(S^{*}\right)$, we can determine in polynomial time the value of $c_{[2]}(S)$ for any solution $S$ satisfying the following criteria, or deduce that no such solution exists:

(i) $S$ is e-critical.

(ii) $c_{[2]}(S)<z\left(S^{*}\right)$.

(iii) $\nexists S^{\prime} \in \mathcal{S}$ with $c_{[2]}\left(S^{\prime}\right)<c_{[2]}(S)$ satisfying (i) and (ii).

Proof. Since $\operatorname{OPT}(\Pi) \in \mathcal{P}$, we can calculate $z\left(S^{*}\right)$ in polynomial time. Let us convert the instance $\pi$ to the instance $\pi_{T}$ as follows: $G \leftarrow G \backslash\{e\}$ and $S \leftarrow S \backslash\{e\}$ for every $S \in \mathcal{S}$. This conversion only affects the cost of $e$-critical solutions satisfying $S \backslash\{e\} \neq \emptyset$, each of which has now a cost equal 
to the cost of its second largest element. If a solution satisfies $S \backslash\{e\}=\emptyset$, then its cost becomes infinite after the conversion.

Let us now apply Algorithm $\mathcal{A}$ to $\pi_{T}$. If the objective value of the output is equal to that of the original problem, then it is obvious that no solution satisfying the three criteria exists. On the other hand, if the objective value of the optimal solution to $\pi_{T}$ is lower, then the new objective value is the required output, since it is the lowest $c_{[2]}(\cdot)$ value among all solutions satisfying conditions (i) and (ii) in $\pi$.

The method mentioned above involves invoking Algorithm $\mathcal{A}$ twice, and transforming a problem instance. Since all the operations are polynomial (we do not explicitly transform individual solutions) the method itself is polynomial.

\section{Theorem 2. Let $\varepsilon>0, \Pi=(G, \mathcal{S}, z)$ be a min-max $C O P$. Then}

$$
\operatorname{OPT}(\Pi) \in \mathcal{P} \Rightarrow \operatorname{SA} \varepsilon(\Pi) \in \mathcal{P} .
$$

Proof. Since $S^{\varepsilon}$ is known, we can predict its behavior when $c_{e}$ changes. Also since $\operatorname{OPT}(\Pi) \in \mathcal{P}$, an optimal solution $S^{*}$ to an instance $\pi$ of $\Pi$ can be calculated in polynomial time.

We will first show that if $\mathrm{OPT}(\Pi) \in \mathcal{P}$, then given an $\varepsilon$-optimal solution $S^{\varepsilon}$ and $e \in G, \beta_{e}$ can be calculated in polynomial time. If $e \notin S^{*}$, then the optimal objective value is not affected by an increase in $c_{e}$. If $e \in S^{*}$, the optimal objective value remains $z\left(S^{*}\right)$ until $c_{e}$ exceeds $z\left(S^{e}\right)$. After that, the new optimal objective value remains constant at $z\left(S^{e}\right)$. So we see that we can deduce the response of the optimal objective value to changes in $c_{e}$. We know that $z\left(S^{e}\right)$ can be calculated in polynomial time. Since we know the responses of both $S^{\varepsilon}$ and the optimal objective value to changes in $c_{e}$ in polynomial time, $\beta_{e}$ can be calculated in polynomial time.

Finally we show that if $\mathrm{OPT}(\Pi) \in \mathcal{P}$, then given an $\varepsilon$-optimal solution $S^{\varepsilon}$ and $e \in G, \alpha_{e}$ can be calculated in polynomial time. If $c_{e}$ decreases, then $S^{*}$ can become suboptimal only if $c_{e}>z\left(S^{*}\right)$, and there exists an $e$-critical solution satisfying the three conditions in Lemma 1 . In that case, the optimal objective value remains $z\left(S^{*}\right)$ until $c_{e}$ reduces to $z\left(S^{*}\right)$, then becomes $c_{e}$ until $c_{e}$ reduces to $c_{[2]}(S)$ for a solution $S$ satisfying the three conditions in Lemma 1 , and then remains constant at $c_{[2]}(S)$. According to Lemma 1, we can check for the existence of such a solution $S$ and find $c_{[2]}(S)$ if it exists, in polynomial time. Therefore we can predict the response of the optimal objective value to changes in $c_{e}$. Since we know the responses of both $S^{\varepsilon}$ and the optimal objective value to changes in $c_{e}$ in polynomial time, $\alpha_{e}$ can be calculated in polynomial time.

In the remainder of the paper, we assume that $\mathrm{SA} \varepsilon(\Pi) \in \mathcal{P}$ and make the further assumption that $\mathrm{OPT} \varepsilon(\Pi) \in \mathcal{P}$. Under these assumptions we 
show that an optimal solution to any instance $\pi$ of $\Pi$ can be calculated in polynomial time.

The following two lemmas provide a polynomial time characterization of the objective value of an optimal solution.

Lemma 3. Given $\varepsilon>0$, an $\varepsilon$-optimal solution $S^{\varepsilon}$ to an instance $\pi \in \Pi$, and an element $e \in S^{\varepsilon}$ such that $z\left(S^{\varepsilon}\right)=c_{e}$, we have: $\beta_{e} \geq \varepsilon z\left(S^{\varepsilon}\right) \Leftrightarrow$ $z\left(S^{*}\right)=z\left(S^{\varepsilon}\right)$ for any optimal solution $S^{*}$ to $\pi$.

Proof. $(\Rightarrow)$ Assume to the contrary that there exists an optimal solution $S_{1}^{*}$ with $z\left(S_{1}^{*}\right)<z\left(S^{\varepsilon}\right)$. Then $e \notin S_{1}^{*}$, and the value of $z\left(S_{1}^{*}\right)$ is not affected by an increase in $c_{e}$ while that of $S^{\varepsilon}$ increases with a slope of 1 .

Hence $\beta_{e}=(1+\varepsilon) z\left(S^{*}\right)-z\left(S^{\varepsilon}\right)<(1+\varepsilon) z\left(S^{\varepsilon}\right)-z\left(S^{\varepsilon}\right)=\varepsilon z\left(S^{\varepsilon}\right)$, which is a contradiction.

$(\Leftarrow)$ We distinguish between the following two cases:

1. $e \in S^{*}$ for all $S^{*}$.

2. There exists $S^{*}$ such that $e \notin S^{*}$.

In case 1 , if the value of $c_{e}$ increases, both $z\left(S^{\varepsilon}\right)$ and $z\left(S^{*}\right)$ increase with a slope of 1 until they reach the value $z\left(S^{e}\right)$. After that, $z\left(S^{\varepsilon}\right)$ keeps increasing with slope 1 , but $z\left(S^{*}\right)$ remains constant at $z\left(S^{e}\right)$. It follows that $\beta_{e}=$ $\left(z\left(S^{e}\right)-z\left(S^{*}\right)\right)+\varepsilon z\left(S^{e}\right) \geq \varepsilon z\left(S^{\varepsilon}\right)$.

In case 2 , the objective value of the optimal solution is not affected by an increase in the value of $c_{e}$ but $z\left(S^{\varepsilon}\right)$ increases with a slope of 1 . So clearly $\beta_{e}=\varepsilon z\left(S^{\varepsilon}\right)$.

Lemma 4. Given $\varepsilon>0$, an $\varepsilon$-optimal solution $S^{\varepsilon}$ to an instance $\pi \in \Pi$, and an element $e \in S^{\varepsilon}$ such that $z\left(S^{\varepsilon}\right)=c_{e}$, we have: $\beta_{e}<\varepsilon z\left(S^{\varepsilon}\right) \Rightarrow$ $z\left(S^{*}\right)=\left(c_{e}+\beta_{e}\right) /(1+\varepsilon)$ for any optimal solution $S^{*}$ to $\pi$.

Proof. It follows from Lemma 3 that $\beta_{e}<\varepsilon z\left(S^{\varepsilon}\right) \Rightarrow z\left(S^{*}\right)<z\left(S^{\varepsilon}\right)$. Therefore $e \notin S^{*}$, which implies that the objective value of the optimal solution is not affected by an increase in the value of $c_{e}$ but $z\left(S^{\varepsilon}\right)$ increases with a slope of 1 . So $z\left(S^{\varepsilon}\right)+\beta_{e}=c_{e}+\beta_{e}=(1+\varepsilon) z\left(S^{*}\right)$. The result follows.

Theorem 5. Let $\varepsilon>0$ and $\Pi=(G, \mathcal{S}, z)$ be a min-max COP. Then

$$
\mathrm{SA} \varepsilon(\Pi), \operatorname{OPT} \varepsilon(\Pi) \in \mathcal{P} \Rightarrow \operatorname{OPT}(\Pi) \in \mathcal{P} .
$$

Proof. From Lemmas 3 and 4 we know that if $\operatorname{SA} \varepsilon(\Pi), \operatorname{OPT} \varepsilon(\Pi)$ $\in \mathcal{P}$, then the objective value of an optimal solution can be calculated in polynomial time. This is equivalent to saying that the evaluation version of $\Pi$ is polynomially solvable.

It is common knowledge that if the evaluation version of a COP is polynomially solvable, so is the optimization version. For the sake of completeness, we present the following Algorithm $\mathcal{B}$ that generates an optimal solution 
to an instance $\pi$ of $\Pi$, given a polynomial time algorithm to calculate an $\varepsilon$-optimal solution to $\pi$.

\section{Algorithm $\mathcal{B}$}

Input: An instance $\pi$ of $\Pi$, an algorithm to calculate an $\varepsilon$-optimal solution to $\pi$.

Output: An optimal solution to $\pi$.

begin

$S \leftarrow \emptyset ;$

Obtain an $\varepsilon$-optimal solution $S^{\varepsilon}$ to $\pi$;

Obtain $z^{*}$, the optimal objective value of $\pi$;

if $z^{*}=z\left(S^{\varepsilon}\right)$ then

Return $S^{\varepsilon}$ and stop;

Arrange elements $e \in G$ in non-increasing order of $c_{e}$ values;

/* the elements will be chosen in this order in the following for loop.*/

for each $e \in G$ do

begin

Apply $T: c_{e} \leftarrow \infty$;

Obtain an $\varepsilon$-optimal solution $S^{\varepsilon}$ to $\pi_{T}$;

Obtain $z^{T}$, the optimal objective value of $\pi_{T}$;

if $z^{T}<z^{*}$ then

begin

$S \leftarrow S \cup\{e\} ;$

Restore the value of $c_{e}$;

end

end

Return $S$;

end.

It is trivial to see that Algorithm $\mathcal{B}$ is correct. Apart from a (polynomial) sorting operation, the algorithm calculates $\varepsilon$-optimal solutions and uses them to calculate the optimal objective value $\mathcal{O}(|G|)$ times. According to Lemmas 3 and 4, the latter operation is polynomial time. Hence Algorithm $\mathcal{B}$ is also a polynomial algorithm.

In Theorem 2 we established that the sensitivity analysis problem for COPs with min-max objective functions can be done in polynomial time if the original COP can be solved in polynomial time. In Theorem 5 we showed that if the sensitivity analysis problem for a COP is polynomially solvable, so is the original COP, under the additional weak assumption that an $\varepsilon$-optimal solution can be obtained in polynomial time. Hence we deduce that the sensitivity analysis problem for $\varepsilon$-optimal solutions to combinatorial problems with min-max objectives is as difficult as the original problems themselves. 
The results in this paper are interesting for several reasons. From a practical point of view, they immediately apply to a wide variety of problems, for example, bottleneck traveling salesperson problems, scheduling problems, packing problems, etc. From the theoretical point of view, the results presented here complement those on min-sum problems and on optimal solutions to both min-sum and min-max problems (refer, for example, to [17] and [9]). Finally, the techniques used extend those in [17] and [9] in a non-trivial manner.

\section{References}

[1] C. E. Blair, Sensitivity analysis for knapsack problems: A negative result, Discrete Appl. Math. 81 (1998), 133-139.

[2] C. E. Blair and R. G. Jereslow, Computational complexity of some problems in parametric discrete programming I, Math. Oper. Res. 11 (1986), 241-260.

[3] P. J. Cartensen, Complexity of some parametric integer and network programming problems, Math. Programming 26 (1983), 64-75.

[4] N. Chakravarti and A. P. M. Wagelmans, Calculation of stability radii for combinatorial optimization problems, Oper. Res. Lett. 23 (1998), 1-7.

[5] W. Cook, A. M. H. Gerards, A. Schrijver, and É. Tardos, Sensitivity theorems in integer linear programming, Math. Programming 34 (1986), 251-264.

[6] E. N. Gordeev and V. K. Leontev, Stability in bottleneck problems, Comput. Math. Math. Phys. 20 (1981), 275-280.

[7] E. N. Gordeev, V. K. Leontev, and I. Kh. Sigal, Computational algorithms for finding the radius of stability in problems of choice, ibid. 23 (1983), 973-979.

[8] H. J. Greenberg, An annotated bibliography for post-solution analysis in mixed integer programming and combinatorial optimization, in: Advances in Computational and Stochastic Optimization, Logic Programming, and Heuristic Search, D. L. Woodruff (ed.), Kluwer, 1998, 97-148.

[9] S. van Hoesel and A. P. M. Wagelmans, On the complexity of postoptimality analysis of 0/1 programs, Discrete Appl. Math. 91 (1999), 251-263.

[10] M. Libura, Sensitivity analysis for minimum Hamiltonian path and traveling salesman problems, ibid. 30 (1991), 197-211.

[11] - Optimality conditions and sensitivity analysis for combinatorial optimization problems, Control Cybernetics 25 (1996), 1165-1180.

[12] - On accuracy of solutions for discrete optimization problems with perturbed coefficients of the objective function, Ann. Oper. Res. 86 (1999), 53-62.

[13] M. Libura, E. S. van der Poort, G. Sierksma, and J. A. A. van der Veen, Stability aspects of the traveling salesman problems based on k-best solutions, Discrete Appl. Math. 87 (1998), 159-185.

[14] R. Nauss, Parametric Integer Programming, Univ. of Missouri Press, Columbia, MO, 1979.

[15] E. S. van der Poort, G. Sierksma, and J. A. A. van der Veen, Determining tolerances for the traveling salesman problem, SOM Report No. 97A27, Univ. of Groningen.

[16] R. Ramaswamy, Sensitivity analysis in combinatorial optimization, doctoral thesis, Indian Institute of Management, Calcutta, 1994. 
[17] R. Ramaswamy, N. Chakravarti, and D. Ghosh, Complexity of determining exact tolerances for min-max combinatorial optimization problems, SOM Report No. 00A22, Univ. of Groningen, 2000, http://www.ub.rug.nl/eldoc/som/a/00A22/00A22.pdf.

[18] Yu. N. Sotskov, Stability of high-speed optimal schedules, Comput. Math. Math. Phys. 29 (1989), 57-63.

[19] - , The stability of the approximate Boolean minimization of a linear form, ibid. 33 (1993), 699-707.

[20] Yu. N. Sotskov, V. S. Tanaev, and F. Werner, Stability radius of an optimal schedule: A survey and recent developments, in: G. Yu (ed.), Industrial Applications of Combinatorial Optimization, Kluwer, 1998, 72-108.

[21] A. P. M. Wagelmans, Sensitivity analysis in combinatorial optimization, Ph.D. thesis, Econometric Institute, Erasmus Univ., Rotterdam, 1990.

[22] G. J. Woeginger, Sensitivity analysis for knapsack problems: Another negative result, Discrete Appl. Math. 92 (1999), 247-251.

Production \& Quantitative Methods Area

Indian Institute of Management

Vastrapur, Ahmedabad 380015, India

E-mail: diptesh@iimahd.ernet.in
Faculty of Economic Sciences University of Groningen P.O. Box 800

9700AV Groningen, The Netherlands E-mail: G.Sierksma@eco.rug.nl

Received on 15.11.2001;

revised version on 31.10.2002 